\title{
Attend Paid Job
}

National Cancer Institute

\section{Source}

National Cancer Institute. Attend Paid Job. NCI Thesaurus. Code C95551.

The ability to maintain gainful employment. 